http://jmscr.igmpublication.org/home/ ISSN (e)-2347-176x ISSN (p) 2455-0450 crossref DOI: https://dx.doi.org/10.18535/jmscr/v8i9.15

\title{
A Complicated Case of Recurrent Liver Abscess Requiring Right Hepatectomy
}

\author{
Authors
}

\section{Dr Pramod Nichat ${ }^{1}$, Dr Pravin Agarwal $^{2}$, Dr Priya Ahire ${ }^{3}$, Dr Saad Shaikh ${ }^{4}$, Dr Kushagra Rahul ${ }^{5}$, Dr Geoffrey Kharmutee ${ }^{6 *}$, Dr Dinesh Goswami ${ }^{7}$, Dhiraj Kachare ${ }^{8}$ \\ ${ }^{1,2,3}$ Master in Surgery, General Surgery, Grant Government Medical College, Mumbai, Maharashtra, India \\ ${ }_{5,6,7,8}$ Resident under General Surgery, General Surgery, Grant Government Medical College Mumbai \\ Maharashtra India \\ *Corresponding Author \\ Dr Geoffrey Kharmutee}

\begin{abstract}
Abscess of the liver has been described since the time of Hippocrates (400 BCE), with the first published review by Bright appearing in 1936.In 1938, Ochsner's classic review heralded surgical drainage as the definitive therapy, as in most cases the abscess spares the biliary tree ${ }^{[1]}$.

But few of the abscesses are invasive enough to involve the biliary tree where simple drainage can lead to the formation of a biliary fistula ${ }^{[2]}$. Such cases require extensive radiological elucidation and surgical management ${ }^{[2,3]}$.

The authors here present a case of liver abscess which in spite of drainage with USG guided pigtail/aspiration and conservative management progressed into a non healing recurrent abscess involving the right biliary tree requiring right hepatectomy.
\end{abstract}

\section{Introduction}

Liver abscess was regarded a high morbidity disease requiring open surgical drainage, with mortality rates between $9 \%$ and $80 \%^{[3]}$. If untreated, it was uniformly fatal. In the last quarter of a century we have witnessed a major paradigm shift in the management of pyogenic hepatic abscesses, with a concomitant decrease in mortality and the current mortality rate is low, ranging from $4.2 \%$ to $11.7 \%{ }^{[4,5]}$. Advances in diagnostic and interventional radiology over the last three decades have facilitated a minimally invasive approach to management of this condition. In combination with targeted antimicrobial therapy, USG guided drainage techniques now form the mainstay of treatment. However, a small proportion of patients do not respond appropriately to minimally invasive management strategies, it is critical to promptly recognize these patients, for whom traditional open surgical intervention is the definitive treatment. $^{[6]}$

\section{Case Report}

A 31 year old male patient presented in the Out Patient Department with complaints of pain in the right hypochondriac region associated with fever, nausea since 6 days. There was no history of trauma, vomiting, diarrhoea, burning micturition or jaundice. The patient did not have any co 
morbidities or past surgical history. On examination, the general survey of the patient was within normal limits.

Examination of the abdomen revealed that he had tenderness in the right hypochondrium. There was no organomegaly or abdominal lump. The breath sounds were decreased in the right lower zone. Rest of his systemic examination was normal. The patient had a CECT report suggestive of large right lobe liver abscess measuring $2106 \mathrm{cc}$ in volume and involving segment VII and VIII and extending into segment $\mathrm{V}$ and VI(Fig-1). The report was also suggestive of mild right sided pleural effusion.

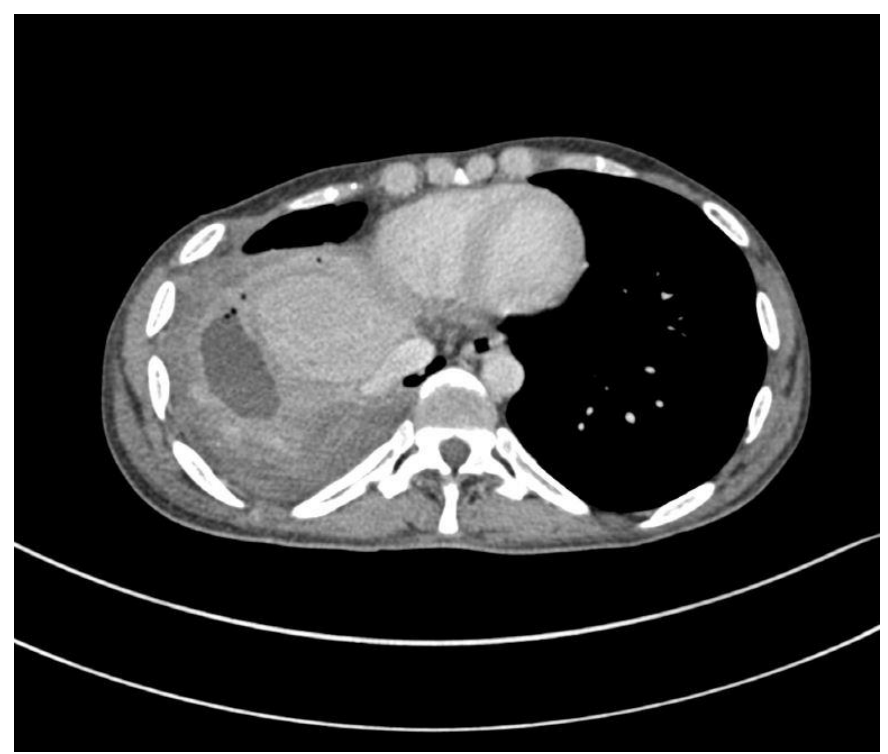

Fig-1: CECT (abdomen) report suggestive of large right lobe liver abscess.

His chest $\mathrm{x}$ ray showed opacification in the right lower lobe with blunting of cost ophrenic angle suggestive of pleural effusion. He was started on broad spectrum intravenous antibiotics, antipyretics and analgesics.

Day 2-On USG, a hypoechoic lesion of approx. volume $166 \mathrm{cc}$ was noted in the segment $\mathrm{V}$ and VIII of liver and another hypoechoic lesion predominantly unliquified of $487 \mathrm{cc}$ was noted in segments VII and VIII of liver and USG guided pigtailing was done to drain the abscess in segments VII and VIII of liver ${ }^{[6]}$.

Day 3-The patient eventually had derranged liver function reports with hypoalbuminemia and developed anasarca. He was resuscitated and injection albumin and diuretics along with high protein diet was given. An intercostal drainage tube insertion was done for right pleural effusion after failing of conservative management and was later on removed after5 days with a review normal chest $\mathrm{x}$-ray.

Day 5-ERCP was done with cholangiogram ${ }^{[7]}$ s/o active bile leak from right anterior biliary radicles, a 7F $\times 12 \mathrm{~cm}$ plastic biliary stent was placed across the leak and free flow of bile was seen , patient was advised immediate post procedure $\mathrm{x}$ ray and USG with LFT after 1 month and stent removal once leak stops.

Day 8- MRCP was done s/o 6.3x4.8x9.1 cm lesion in segment VII/VIII of liver with pigtail catherter in situ, no obvious bile leak or communication with biliary tree at present scan. Mild IHBR dilation in segment VII of liver. ERCP stent in situ.

Day 17-Review USG s/o hypoechoic subdiaphragmatic collection and suprahepatic collection of approx. 20-25 cc with multiple air foci, another hypoechoic lesion with multiple air foci within approx. volume $50 \mathrm{cc}$ is noted in segment VII/VIII of right lobe of liver communicating with subdiaphragmatic liver collection with pigtail tip in situ.

Day 25-Review USG s/o hypoechoic subdiaphragmatic collection and suprahepatic collection of approx. 5-10 cc is noted with multiple air foci. Another hypoechoic lesion with air foci within approx. volume $17 \mathrm{cc}$ noted in the segments VII and VIII of right lobe of lier communicating with subdiaphragmatic liver collection.

Broad spectrum intravenous antibiotics was continued according to drain culture sensitivity and metrodinazole irrigation was done with monitoring of drain output.

The pigtail drain output was decreasing day by day and the pigtail in the subdiaphragmatic and suprahepatic cavity was removed after clamping for 5 days Later on the patient was discharged 
with stable vitals, passed stool and was on full diet.

Day 54-Patient was readmitted with pain in abdomen and low grade fever on USG reports s/ohypoechoic collection partially liquified with multiple air foci of approx. volume 48-50cc noted and immediate USG guided pig tailing was done.

Day 61-Review USG s/o hypoechoic subdiaphragmatic collection and suprahepatic collection volume $20-25 \mathrm{cc}$ [liquified] with pigtail tip in situ.

Day 68-Review USG s/o A residual liver abscess of volume of 20cc [liquified] is noted in the segment VIII of right lobe of liver with pigtail in situ.

Post procedure 12 hourly drain output was monitored and high output bilious fliud was noted on day 10 and was sent for investigations which was confirmed for presence of bile salts.

Day 78-Interventional Radiology reference was done in view of application of cyanoacrylate glue over the leak from the right biliary radicles but was not feasible as it may lead to sclerosis of the whole biliary tree so a repeat ERCP was done with cholangiogram s/o active bile leak from right anterior biliary radicals with sphicterotomy done and a $7 \mathrm{~F} \times 12 \mathrm{~cm}$ plastic biliary stent ${ }^{[8]}$ was replaced with $10 \mathrm{~F} \times 12 \mathrm{~cm}$ to prevent bile leak but post procedure high output bilious fliud was noted from the pigtail drain.

Day 80-MRCP was advised which was s/o intrahepatic billary radicle dilatation in segments V,VII,VIII and communication of anterior segmental bile duct branches with $4.2 \times 2.5 \times 5 \mathrm{~cm}$ in segment VIII of liver extending in the subcapsular space with communication of the main hepatic duct likely forming abscess and normal Common Bile Duct in calibre (Fig-2,3).Patient was contimued with conservative management with double dose of iv injection fluroquinolones and antiprotozoal (Metrodinazole) given for a course of 14 days buthigh output bilious fliud was noted from the pigtail drain. Patient was then planned for Right Hepatectomy ${ }^{[9]}$.

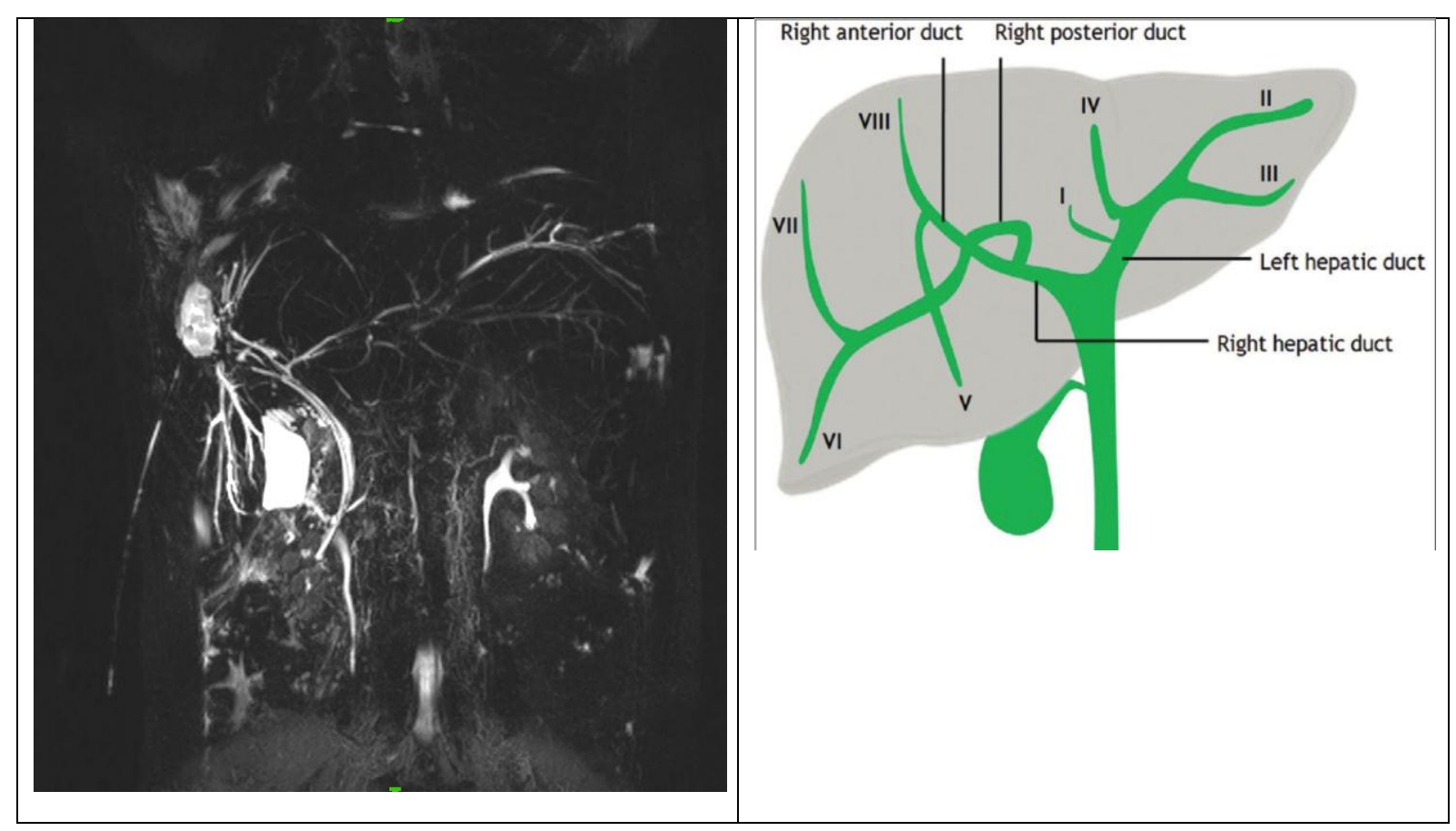

Fig-2,3: (Right) MRCP showing involvement of the anterior bile duct and main hepatic duct with (Left) Anatomy of normal bile duct distribution.

Day 94-Exploratory Laparotomy and cholecystectomy was done. Portal vein and right hepatic artery was identified and dissected, posterior adhesions of the liver were dissected 
with blunt dissection and torrential bleeding was noted from the raw surface. Packing of the liver was done and pressure applied. After few minutes dissection started again and abscess cavity identified, opened and debrided. Pigtail seen once the cavity was opened. Torrential bleeding occurred once again and hepatic packing with mops done and intra operative multiple blood and blood products transfusion was given. In view of massive blood loss and hypotension, immediate intervention was taken as Damage Control Surgery Procedure ${ }^{[10]}$. Patient was shifted to intensive care unit and was intubated and on inotropes. Patient was thoroughly monitored for 48 hours and was planned for re-exploration.

Day 96-Re-exploratory Laparotomy with removal of perihepatic packing was done. Liver was dissected posteriorly off the Inferior vena cava. Three rents over the Inferior vena cava and bleeding from the liver surface was identified and repaired. Right hepatic vein identified and clamped. Right Hepatectomy was done using Cavitron Ultrasonic Surgical Aspirator $(\mathrm{CUSA})^{[11]}$. Hemostasis was achieved. Patient was kept under intensive care for $72 \mathrm{hrs}$ on inotropes and injection albumin which was tapered and later stopped. Blood and blood products was transfused as per hemoglobin and coagulation factors levels respectively.

Day 101-Post operative day 5,he eventually developed fever. Post operative cause of fever was ruled out and ultrasonography was done which was s/o 60-70cc collection over the subhepatic space.USG guided pigtailing was done and drain fluid was sent for culture senstivity. Broad spectrum antibiotics was given as per drain fluid culture sensitvity.

Day 119-As drain output was minimal, a review ultrasonography was done s/o a small $1.0 \times 1.4 \mathrm{~cm}$ subdiaphragmatic collection with pig tail insitu. Eventually, pigtail was removed and oral antibiotics/antiprotozoal was continued. Patient was later on discharged with stable vitals, passed stools and on full diet and was advise to follow up.

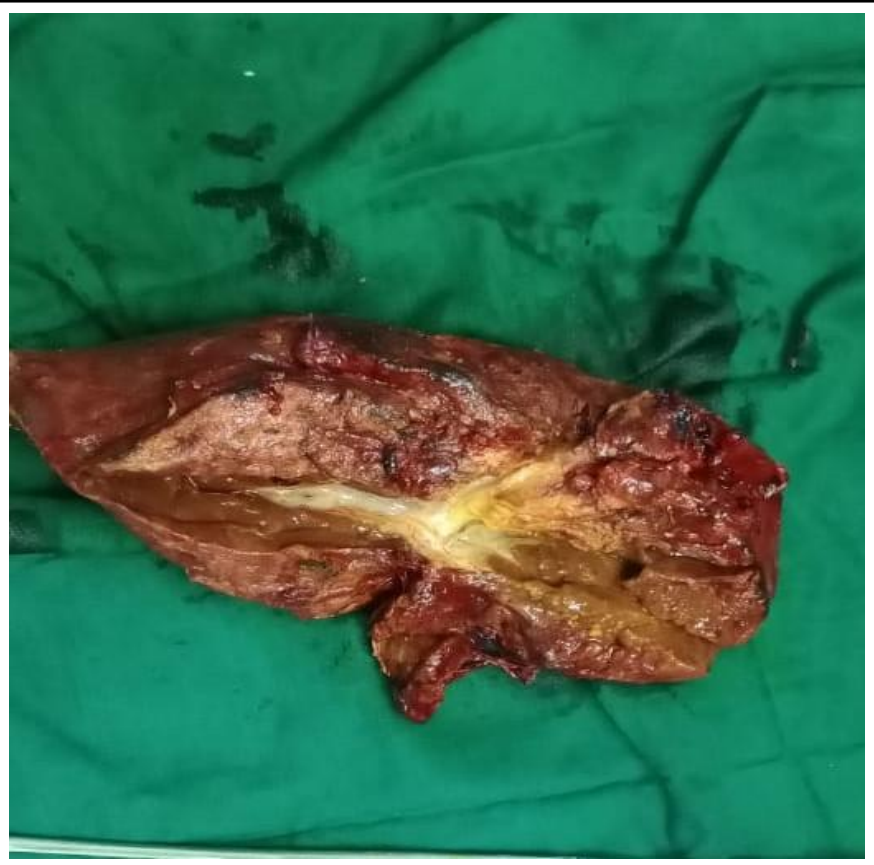

Fig-4: Right lobe of the Liver with parenchymal necrosis.

\section{Discussion}

There are many causes of liver abscess and thus the size and location of the abscesses determine the type of management. Non-invasive measures obviously taken priority ${ }^{[12]}$. Empiric antibiotic coverage is essential. Duration of treatment varies but is usually from two to six weeks. After initial intravenous treatment, orals can be used safely in most cases to complete the course ${ }^{[13]}$. Antibiotics can be given post-drainage to increase culture yield for proper treatment.Currently, USG guided drainage and antibiotics suffice for most liver abscesses but non healing recurrent abscess involving the biliary tree which can prolonged hospital stay, readmission and complications requiring right hepatectomy should be taken in account. The discovery of new procedures and techniques, life-threatening and high mortality rates decreases ${ }^{[14]}$.

\section{Conclusion}

Liver abscess reoccurrence in patients who present with a biliary tract disease can lead to non healing complications and prolonged illness. Immediate and definitive intervention should be taken with co relation of clinical examination and a series of investigations. With new techniques available for 
drainage like USG guided drainage and Pigtailing, antibiotics specific for appropriate organisms, liver abscesses can have a much better prognosis now. Newer procedures like Cavitron Ultrasonic Surgical Aspirator lessens blood loss during the procedure and precisely maintains integrity of critical blood vessels without damage and ensures safety without sacrificing speed and need for postoperative blood transfusion and complications.

\section{References}

1. Chiu CT, Lin DY, Wu CS, Chang-Chien CS, Sheen IS, Liaw YF. A clinical study on pyogenic liver abscess. Taiwan Yi Xue Hui Za Zhi. 1987;86:405-412.

2. Branum GD, Tyson GS, Branum MA, Meyers WC. Hepatic abscess. Changes in etiology, diagnosis, and management. Ann Surg. 1990;212(6):655-662. doi: 10.1097/00000658-199012000-00002.

3. Rahimian J, Wilson T, Oram V, Holzman RS. Pyogenic liver abscess: recent trends in etiology and mortality. Clin Infect Dis. 2004;39(11):1654-1659. doi: $10.1086 / 425616$.

4. Hope WW, Vrochides DV, Newcomb WL, et al. Optimal treatment of hepatic abscess. Am Surg. 2008;74(2):178-182.

5. Lee KT, Wong SR, Sheen PC. Pyogenic liver abscess: an audit of 10 years' experience and analysis of risk factors. Dig Surg. 2001;18(6):459-465.

doi: 10.1159/000050194.

6. Branum GD, Tyson GS, Branum MA, Meyers WC. Hepatic abscess. Changes in etiology, diagnosis, and management. Ann Surg. 1990;212:655-662.

7. Baek SY, Lee MG, Cho KS, Lee SC, Sung KB, Auh YH. Therapeutic percutaneous aspiration of hepatic abscesses: effectiveness in 25 patients. AJR. 1993;160:799-802.
8. Endoscopic

Retrograde

Cholangiopancreatography

(ERCP)

Technique Updated: May 30,

2019 Author: Ahmad Malas, MD; Chief

Editor: Kurt E Roberts, MD [medscape.com/article/1829797.

9. Biliary Stenting-Updated: Sep 30, 2019Author: Philip L Johnson, MD; Chief Editor: Kyung J Cho, MD, FACR, FSIR [medscape.com/article/1828072overview]

10. Blumgart LH, Belghiti J. Surgery of the liver, biliary tract, and pancreas. 4th ed. Philadelphia, PA: Saunders Elsevier, 2007.

11. Sharp KW, Locicero RJ. Abdominal packing for surgically uncontrollable haemorrhage. Ann Surg. 1992;215:467475.

12. Rau HG, Duessel AP, Wurzbacher S. The use of water-jet dissection in open and laparoscopic liver resection. HPB (Oxford). 2008;10:275-280.

13. Bertel CK, van Heerden JA, Sheedy PF., 2nd Treatment of pyogenic hepatic abscesses. Surgical vs percutaneous drainage. Arch Surg. 1986;121:554-558.

14. Stain SC, Yellin AE, Donovan AJ, Brien HW. Pyogenic liver abscess. Modern treatment. Arch Surg. 1991;126:991-996.

15. Farges O, Leese T, Bismuth H. Pyogenic liver abscess: an improvement in prognosis. Br J Surg. 1988;75(9):862-865. doi: 10.1002/bjs.1800750910. 